\title{
Ear Reconstruction Utilizing the Subcutaneous Island Pedicle Graft (Flip-Flop) Flap
}

\author{
Darrell J. Fader, MD*,† and Timothy M. Johnson, MD*,†,‡ \\ Departments of *Dermatology, ${ }^{\dagger}$ Otolaryngology, and ${ }^{\ddagger}$ Surgery (Division of Plastic Surgery), University of Michigan \\ Medical and Comprehensive Cancer Center, Ann Arbor, Michigan
}

SOFT TISSUE RECONSTRUCTION of the conchal aspects of the external ear includes a variety of approaches, including granulation, graft, or local skin flap. We describe our experience with the single-stage subcutaneous island pedicle graft (flip-flop) flap for reconstruction of defects with a significant amount of exposed cartilage involving primarily the concha, antitragus, antihelix, and external auditory meatus (EAM).

\section{Patients}

The subcutaneous island pedicle graft flap was utilized in 13 patients immediately following Mohs excision of basal cell carcinoma (12) or Bowen's disease (1). Defect size ranged from $0.7 \times 0.8 \mathrm{~cm}$ to $3.5 \times 4.2 \mathrm{~cm}$ (mean $1.9 \times 2.3 \mathrm{~cm})$. All defects included loss of fullthickness skin with exposed cartilage. Seven defects were located in the concha (two in the cymba, five in the cavum), three on the EAM, two on the antihelix, and one on the antritragus.

Ten of the 13 patients underwent excision of the tumor on the ear creating a primary ear defect. In three patients, the primary defect resulted from excision of the tumor located on the sebaceous nasal tip. In these three patients, a full thickness skin graft was harvested from the conchal bowl due to excellent tissue match with conchal skin with sebaceous quality. Perichondrium was included on each graft due to the depth of the nasal defect. Repair of the secondary conchal ear defect was then performed utilizing the subcutaneous island pedicle graft flap.

\section{Operative Technique}

A telfa template is formed and cut to match the size and shape of the auricular defect. The template is placed and outlined in the postauricular sulcus, with an effort to utilize more retroauricular and mastoid skin than posterior ear skin. This will minimize ear pinning when closing the secondary defect. For conchal defects, residual exposed cartilage is excised. Inci-

\footnotetext{
Address correspondence and reprint requests to: Darrell J. Fader, MD, University of Michigan, Department of Dermatology, 1910 Taubman Center, Box 0314, Ann Arbor, MI 48109-0314.
}

sions are made along the outlined flap, and all aspects of the pedicle flap and defect are undermined by sharp or blunt dissection. A subcutaneous vascular pedicle is preserved at or close to the center of the flap. With a hook or forceps, the island pedicle graft flap is tunneled from the postauricular sulcus to the anterior conchal defect. The flap is trimmed of excess adipose tissue and sutured into the defect in a standard layered or simple fashion. The postauricular secondary defect is closed primarily. The operative technique is illustrated in Figures 1-4. The great auricular and lesser occipital nerves provide sensory innervation to the helix, antihelix, lobule, and postauricular skin. Care should be taken to try to identify and preserve these nerves to avoid prolonged numbness. When closing larger secondary postauricular defects, pinning of the ear may be minimized by designing a local transposition flap (rhombic, z-plasty) in the postauricular sulcus at the time of initial reconstruction. Alternatively, a z-plasty may be undertaken several months later if necessary.

\section{Results}

Infection or flap necrosis did not occur in our series. Cosmetic and functional outcomes were considered excellent by patient and surgeon. Six weeks postoperatively, one patient observed a minor pinning of the ear back toward the scalp, yet this improved with time and a revision was not sought. Another patient early in our series developed a mild trap door phenomenon which resolved with intralesional steroid injections and massage.

\section{Discussion}

Numerous reconstructive options are available to the cutaneous surgeon for the repair of conchal, antitragus, antihelix, and EAM defects. Second intention healing may be ideal, particularly when perichondrium is intact. In the absence of perichondrium, exposed cartilage left to granulate may result in painful, chronic chondritis and increase the risk of infection in an area already at higher risk. ${ }^{1}$ Healing is also relatively slow for significant defects. Punch excision of cartilage decreases healing time by allowing granulating tissue to fill the wound from both the center and 

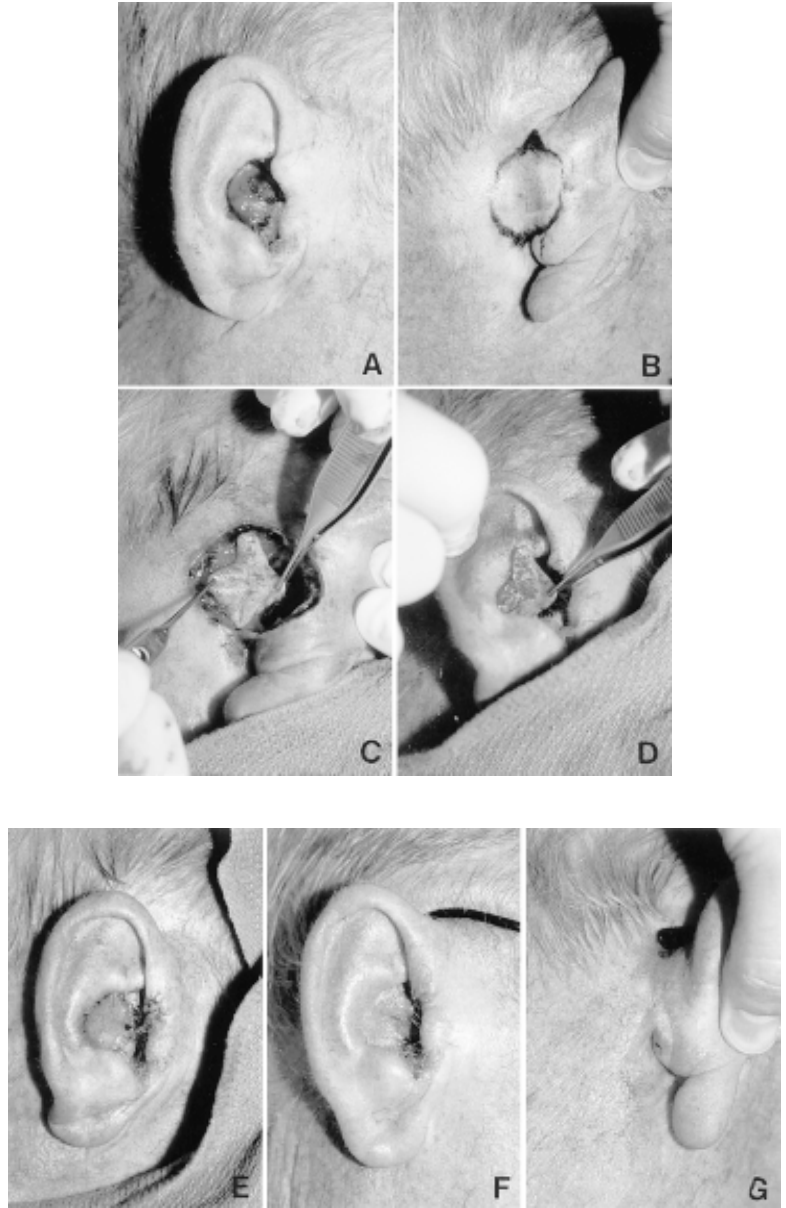

Figure 1. A. Following Mohs excision of a basal cell carcinoma, a full thickness soft tissue defect comprises most of the conchal bowl. B. A template of the defect is outlined in the postauricular sulcus. C. After exposed cartilage is removed, the flap is incised, preserving a central subcutaneous vascular pedicle. D. The flap is pulled through to cover the anterior defect and E. it is sutured in place. The secondary postauricular defect is closed primarily. F and G. Two months later, an excellent cosmetic outcome is achieved.

peripheral edges of the defect. A full- or split-thickness skin graft may be useful in this area. Prior removal of exposed cartilage will enhance graft survival. ${ }^{2}$ Structural integrity following removal of conchal cartilage is insignificant. However, removal of non-conchal cartilage may be problematic especially for large defects extending into the scaphoid fossa. Transposition flaps from the preauricular region may also be appropriate for select conchal, antitragus, antihelix, and EAM defects.

The subcutaneous island pedicle graft (flip-flop) flap offers a simple, one-stage method for repairing anterior ear defects involving the concha, antitragus, antihelix, and EAM. ${ }^{3-5}$ The rich vascular supply from branches of the superficial temporal artery and posterior auricular artery minimize flap necrosis as long as a central pedicle is maintained. 6 Aggressively thinning the circumferential margins of the flap without vascular com-
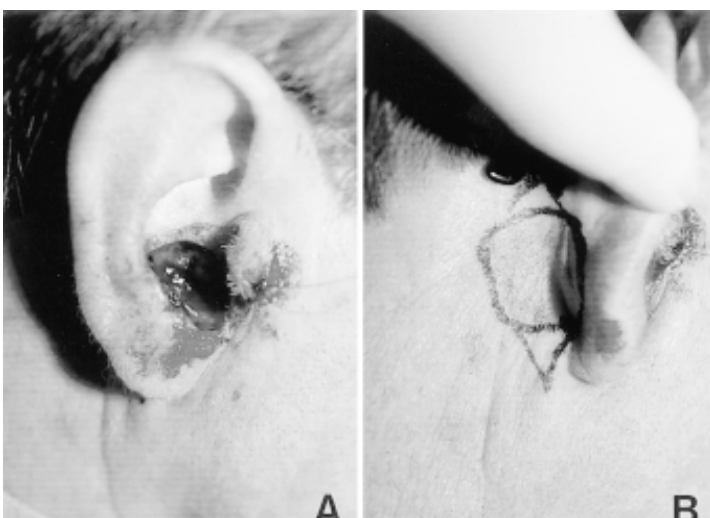

A

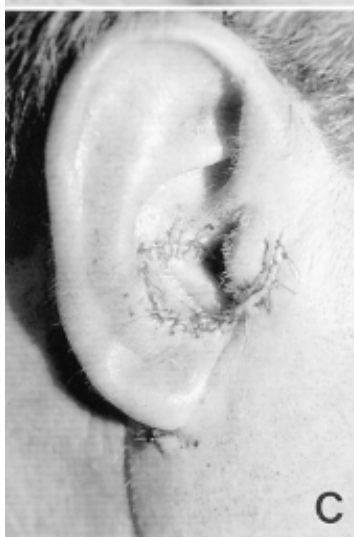

B

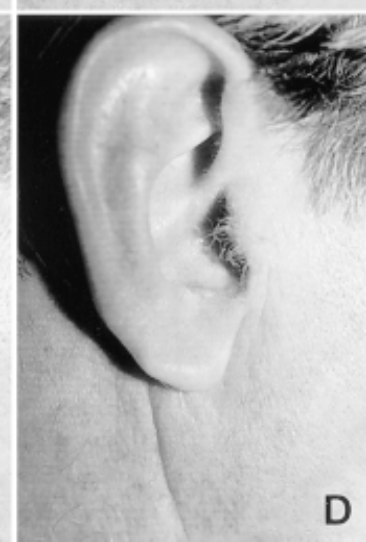

Figure 2. A. A $1.6 \times 2.0 \mathrm{~cm}$ defect of the lower conchal bowl and external auditory meatus. B. The defect is closed with skin recruited more from the retroauricular than postauricular area to minimize ear pinning. C. The flip-flop flap is sutured into place. D. Follow-up occurs at 10 months.

promise may be performed for contour. The cosmetic result may be enhanced with sufficient undermining of the defect to theoretically minimize the trap door phenomenon, present in one of our earlier subjects.

A traditional objection to the subcutaneous island pedicle graft flap is the risk of pinning the ear back to the scalp, particularly with larger flaps in patients with protrusive ears. In our experience, such pinning
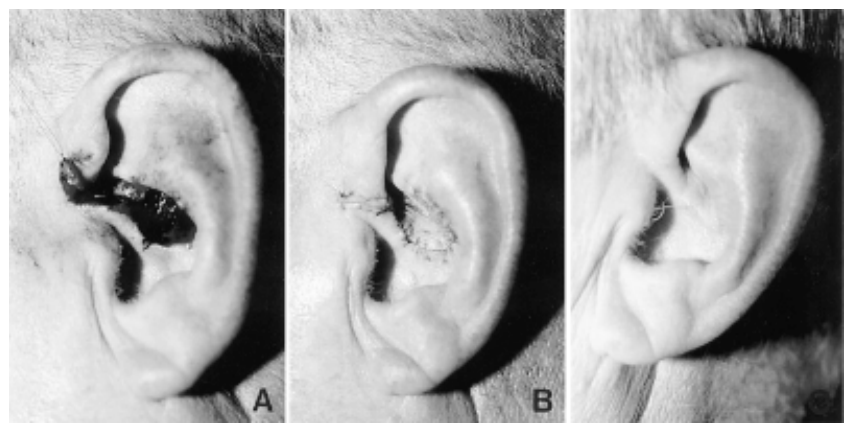

Figure 3. A defect of the superior conchal bowl required a releasing incision across the helical crus for enhanced access to the anterior conchal cymba. B. Both flap and releasing incision are sutured. D. The result is seen two months later. 


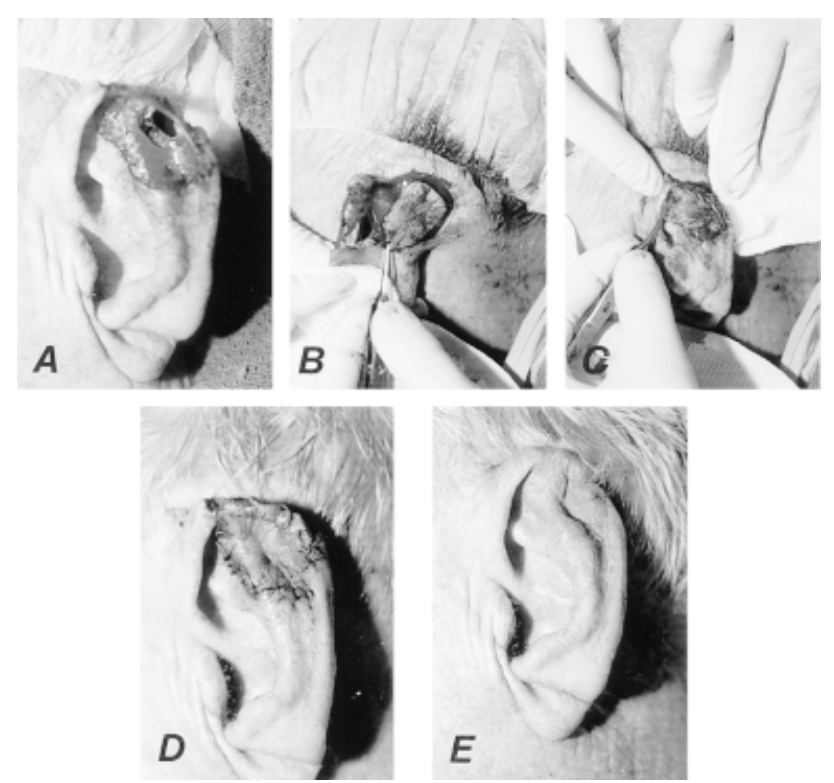

Figure 4. A. Mohs removal of an aggressive basal cell carcinoma has left a $3.5 \times 4.2$ defect of the antihelix and helical rim with a small through-and-through defect. Reconstructive options could include a large skin graft or a two-stage retroauricular interpolation flap. B and C. A flip-flop flap is pulled through the existing cartilage defect, maximizing the structural integrity of the reconstruction. D. Bolster sutures are employed to re-create a helical rim. E. Structural support and helix/antihelix contour are preserved at 6 weeks. has proven temporary, improving within 2 to 3 months. Moreover, design of the flap to minimize the use of auricular skin in favor of scalp skin may circumvent this problem. Consequently, scalp skin is advanced into the postauricular sulcus to close the secondary defect with minimal or no ear pinning. Nevertheless, patients with larger defects $(>3 \mathrm{~cm})$ and/or protrusive ears should be advised regarding the possible need for subsequent revision. A retroauricular transposition flap (rhombic or z-plasty) may then be employed to ameliorate excessive ear pinning.

\section{References}

1. Futoryan T, Grande D. Postoperative wound infection rates in dermatologic surgery. Dermatol Surg 1995;21:509-14.

2. Mellette JR, Swinehart JM. Cartilage removal prior to skin grafting in the triangular fossa, antihelix, and concha of the ear. J Dermatol Surg Oncol 1990;16:1102-5.

3. Masson JK. Subcutaneous pedicle flap for reconstruction of the anterior surface of the external ear. In: Strauch B, Vasconez LO, HallFindlay EJ, eds. Grabb's Encylopedia of Flaps. Volume I Head and Neck. Boston: Little Brown and Co., 1990:309-13.

4. Masson JK. A simple island flap for reconstruction of concha-helix defects. Br J Plast Surg 1972;25:399.

5. Humpreys TR, Goldberg LH, Wiemer DR. The postauricular (revolving door) island pedicle flap revisited. Dermatol Surg 1996;22: 148-50.

6. Salasche SJ, Bernstein G, Senkasik M. The ear. In: Regional Anatomy III: The Ear. In: Surgical Anatomy of the Skin. Norwalk, CT: Appleton and Lange, 1988:220. 